\title{
Qualitative Analysis of a Retarded Mathematical Framework with Applications to Living Systems
}

\author{
Carlo Bianca, ${ }^{1}$ Massimiliano Ferrara, ${ }^{2}$ and Luca Guerrini ${ }^{3}$ \\ ${ }^{1}$ Dipartimento di Scienze Matematiche, Politecnico, Corso Duca degli Abruzzi 24, 10129 Torino, Italy \\ ${ }^{2}$ Department of Law and Economics, University Mediterranea of Reggio Calabria and CRIOS, University Bocconi of Milan, \\ Via dei Bianchi 2, 89127 Reggio Calabria, Italy \\ ${ }^{3}$ Department of Management, Polytechnic University of Marche, 60121 Ancona, Italy
}

Correspondence should be addressed to Carlo Bianca; carlo.bianca@polito.it

Received 3 September 2013; Accepted 12 November 2013

Academic Editor: Constantin Udriste

Copyright $\odot 2013$ Carlo Bianca et al. This is an open access article distributed under the Creative Commons Attribution License, which permits unrestricted use, distribution, and reproduction in any medium, provided the original work is properly cited.

This paper deals with the derivation and the mathematical analysis of an autonomous and nonlinear ordinary differential-based framework. Specifically, the mathematical framework consists of a system of two ordinary differential equations: a logistic equation with a time lag and an equation for the carrying capacity that is assumed here to be time dependent. The qualitative analysis refers to the stability analysis of the coexistence equilibrium and to the derivation of sufficient conditions for the existence of Hopf bifurcations. The results are of great interest in living systems, including biological and economic systems.

\section{Introduction}

In the last three decades, the mathematical modeling of living systems has gained much attention and different mathematical methods have been developed in an attempt to obtain a mathematical theory. Living systems, differently from physical systems, require more attention and a different mathematical treatment depending on the system under investigation.

Different mathematical and also computational frameworks have been proposed whose difficulty is strictly related to the phenomena of the system that has to be modeled. Indeed, if we are only interested in the time evolution of the density of the elements in the system (no spatial dynamics), the framework of the ordinary differential equations can be employed; see the review paper [1]. It is worth stressing that this framework requires the definition of a differential equation for each element of the system; therefore, if the number of elements of the system is very large, the applicability of this framework may be unfeasible. When the latter occurs, a continuum mechanics approach can be performed; this approach consists in deriving mass, momentum, and energy conservation equations by phenomenological models; see the book $[2,3]$. The description is obtained by means of suitable partial differential equations. An intermediate approach is that proposed by the kinetic theory. This approach allows the derivation of integrodifferential equations by considering the role of the interactions that can be conservative, nonconservative, and mutative; see the recent review paper [4].

The time evolution of the elements of the system modeled with the previously mentioned mathematical frameworks has in common the characteristic that the description at a time $t$ depends on the description at the same time $t$. This is an approximation of the reality because the phenomena that occur at a time $t$ are strictly related to the behavior of the system at a previous time $t-\tau$, where $\tau$ is a time lag. Therefore, recently the time delay has been inserted into mathematical models for the biological and economical systems, especially in the ordinary differential equations-based models; see, among others, the review paper [5] and papers [6-14].

This paper is devoted to the definition of a mathematical framework that can be proposed for the modeling of living systems, especially of biological and economical nature. The framework proposed in the present paper is based on ordinary differential equations. Specifically, the mathematical framework consists of a system of two nonlinear ordinary differential equations: a logistic equation with a time lag 
(see papers $[15,16])$ and an equation for the carrying capacity that is assumed to be time dependent. The qualitative analysis refers to the stability analysis of the coexistence equilibrium solution and to the derivation of sufficient conditions for the existence of Hopf bifurcations.

The rest of the paper is organized as follows. After this introduction, Section 2 deals with the definition of the assumptions of the system to be modeled and the derivation of the relative model. Section 3 focuses on the qualitative analysis of the framework and specifically is concerned with the coexistence equilibrium solution and the related stability analysis including sufficient conditions for the existence of a Hopf bifurcation. The quality of the Hopf bifurcation, the stability of the bifurcating periodic trajectory, and the relative period are investigated in Section 4. Finally, we conclude the paper and present applications and research perspectives in Section 5.

\section{The Underlying Mathematical Framework}

This section is concerned with the derivation of the mathematical framework that can be proposed for the modeling of biological and economic systems.

Specifically, we consider two cooperating populations whose density is denoted by $\kappa=\kappa(t)$ and $n=n(t)$, respectively. Furthermore, we assume that the population $n$ is defined by a logistic term with time delay $\tau$ such that the carrying capacity is time dependent and coincides with $\kappa$. Let $r$ be the cooperation rate of population $n, c$ the cooperation rate of population $\kappa$, and $\lambda$ the decrease rate of the population $\kappa$. Bearing all the above assumptions in mind, the mathematical models thus reads as follows

$$
\begin{gathered}
\dot{\kappa}(t)=c n(t) \kappa(t)-\lambda \kappa(t), \\
\dot{n}(t)=r \kappa(t) n(t)\left[1-\frac{n(t-\tau)}{\kappa(t)}\right] .
\end{gathered}
$$

The mathematical framework (1) thus consists of a system of autonomous nonlinear ordinary differential equations with a time delay and is characterized by three nonnegative parameters that have specific meanings and can be tuned with empirical data.

It is worth stressing that the mathematical framework (1) summarizes different mathematical models presented in the literature. Indeed, if $\tau=\lambda=0$, we obtain the models analyzed in papers $[17,18]$ where technological innovation is the driving force behind human population growth; see also papers $[19,20]$ where the authors assume a positive feedback between technology and population; for $\tau=0$ and $\lambda=r$, we obtain the model analyzed in paper [21].

\section{Equilibrium Points and Stability Analysis}

This section is devoted to analytical investigations on the existence and stability analysis of the equilibria of the mathematical model (1). The following proposition states the number of nontrivial equilibrium points.

Proposition 1. The mathematical model (1) admits the unique nontrivial equilibrium point $\left(\kappa_{*}, n_{*}\right)=(\lambda / c, \lambda / c)$.
Proof. The proof of this proposition follows by observing that equilibrium points of system (1) correspond to solutions of the algebraic system $\dot{\kappa}=\dot{n}=0$. Since the mathematical model (1) is composed of autonomous differential equations, then the equilibrium points of the system (1) coincide with those of the undelayed counterpart, namely, when $\tau=0$.

Straightforward calculations show that the characteristic equation of the linearized system at $\left(\kappa_{*}, n_{*}\right)$ of the mathematical model (1) model reads as follows:

$$
\xi^{2}+r n_{*} \xi e^{-\xi \tau}-c r n_{*}^{2}=0
$$

where, for notational convenience, we have $n_{*}=\lambda / c$.

The stability analysis of the nontrivial equilibrium $\left(\kappa_{*}, n_{*}\right)$ will be performed by examining first the undelayed system. Thus, if there is no time delay, (2) reads as follows:

$$
\xi^{2}+r n_{*} \xi-c r n_{*}^{2}=0
$$

and the associated eigenvalues are

$$
\xi_{1,2}=\frac{-r n_{*} \pm n_{*} \sqrt{r^{2}+4 c r}}{2} .
$$

Looking to formula (4), we have two real eigenvalues with opposite signs. Then, the equilibrium point $\left(\kappa_{*}, n_{*}\right)$ is a saddle point (system instable).

Suppose now that $\tau>0$. The instability of the equilibrium point may change when the characteristic equation of the system under consideration has zero or a pair of pure imaginary eigenvalues. The former case cannot happen since it occurs if $\xi=0$ in (2) and it is immediately seen that this leads to the contradiction $-\mathrm{crn} n_{*}^{2}=0$. Now, we study when (2) has pure imaginary roots $\xi= \pm i \omega$, where $\omega$ is a positive real number.

Indeed, if $\xi=i \omega, \omega>0$, is a root of (2), then the following identity must be true:

$$
-\omega^{2}+r n_{*} i \omega e^{-i \omega \tau}-c r n_{*}^{2}=0,
$$

and distinguishing between the real and imaginary parts, we obtain the following two equations:

$$
\begin{aligned}
r n_{*} \omega \cos (\omega \tau) & =0 \Longrightarrow \cos (\omega \tau)=0, \\
\omega^{2}+c r n_{*}^{2} & =r n_{*} \omega \sin (\omega \tau) .
\end{aligned}
$$

The solution of (6) is $\omega \tau=\pi / 2(\omega \tau=(3 \pi / 2)$, in particular the other solution has been excluded because it implies the absurd $\left.0<\omega^{2}+c r n_{*}^{2}=r n_{*} \omega(-1)<0\right)$ and

$$
\omega^{2}-r n_{*} \omega+c r n_{*}^{2}=0
$$

whose solutions read as

$$
\omega=\frac{r n_{*} \pm \sqrt{\left(r n_{*}\right)^{2}-4 c r n_{*}^{2}}}{2}=n_{*}\left(\frac{r \pm \sqrt{r^{2}-4 c r}}{2}\right) .
$$

The following lemma holds. 
Lemma 2. Let $r$ be the population growth rate of population $n$ and $c$ the cooperation rate of population $\kappa$.

(1) If $r-4 c<0$ holds true, then (7) does not admit positive roots. Then, there is no stability switch for system (1). Moreover, since $\left(\kappa_{*}, n_{*}\right)$ is unstable when $\tau=0$, it remains unstable for all $\tau>0$.

(2) If $r-4 c \geq 0$ holds true, then (7) has a unique positive root $\omega_{0}$, if $r-4 c=0$, and admits two different positive roots $\omega_{ \pm}$, respectively, if $r-4 c>0$. As $\tau$ increases, stability switches may occur.

It is worth noting that it may be seen easily that the purely imaginary roots $\omega_{0}$ and $\omega_{ \pm}$are simple. Moreover, from (6), we also obtain a sequence of the critical values of $\tau$ defined as follows:

$$
\tau_{j}^{0}=\frac{\pi}{2 \omega_{0}}+\frac{2 \pi j}{\omega_{0}}, \quad \tau_{j}^{ \pm}=\frac{\pi}{2 \omega_{ \pm}}+\frac{2 \pi j}{\omega_{ \pm}} \quad(j=0,1,2, \ldots) .
$$

Lemma 3. Let $r$ be the population growth rate of population $n$ and $c$ the cooperation rate of population $\kappa$.

(1) If $r^{2}-4 c r=0$, then (2) with $\tau=\tau_{j}^{0}$ has a pair of simple pure imaginary roots $\pm i \omega_{0}$.

(2) If $r^{2}-4 c r>0$, then (2) with $\tau=\tau_{j}^{ \pm}$has two pairs of simple pure imaginary roots $\pm i \omega_{ \pm}$.

3.1. Existence of Hopf Bifurcations. In this subsection, sufficient conditions for the existence of a Hopf bifurcation are stated. The preliminary result is contained in the following lemma.

Lemma 4. Let $\xi(\tau)=\nu(\tau)+i \omega(\tau)$ denote the roots of $(2)$ near $\tau=\tau_{*}$ satisfying $\nu\left(\tau_{*}\right)=0, \omega\left(\tau_{*}\right)=\omega_{*}$, where $\tau_{*} \in$ $\left\{\tau_{j}^{0}, \tau_{j}^{ \pm}\right\}(j=0,1,2, \ldots)$ and $\omega\left(\tau_{*}\right) \in\left\{\omega_{0}, \omega_{ \pm}\right\}$, with $\tau_{j}^{0}, \tau_{j}^{ \pm}$and $\omega_{0}, \omega_{ \pm}$being as defined in (8) and (9), respectively.

(1) Let $r-4 c=0$, so that $\omega_{*}=\omega_{0}$. One has $d\left[\operatorname{Re} \xi\left(\tau_{*}\right)\right] / d \tau=0$. Hence, the transversality condition $d\left[\operatorname{Re} \xi\left(\tau_{*}\right)\right] / d \tau \neq 0$ does not hold.

(2) Let $r-4 c>0$.

(a) If $\omega_{*}=\omega_{+}$, then the transversality condition reads

$$
\frac{d\left[\operatorname{Re} \xi\left(\tau_{*}\right)\right]}{d \tau}>0
$$

(b) If $\omega_{*}=\omega_{-}$, then the transversality condition reads

$$
\frac{d\left[\operatorname{Re} \xi\left(\tau_{*}\right)\right]}{d \tau}<0
$$

Proof. Differentiating (2) with respect to $\tau$ and using (2), we get

$$
\frac{d \xi}{d \tau}=\frac{\left(c r n_{*}^{2}-\xi^{2}\right) \xi^{2}}{2 \xi^{2}+\left(c r n_{*}^{2}-\xi^{2}\right)(1-\xi \tau)} .
$$

Substituting $\xi=i \omega_{*}$ into (12), flipping it over, and taking its real part, one has

$$
\left.\operatorname{Re}\left(\frac{d \xi}{d \tau}\right)^{-1}\right|_{\tau=\tau_{*}}=\frac{2}{c r n_{*}^{2}+\omega_{*}^{2}}-\frac{1}{\omega_{*}^{2}} .
$$

Using (7), then (13) reads

$$
\left.\operatorname{Re}\left(\frac{\xi}{d \tau}\right)^{-1}\right|_{\tau=\tau_{*}}=\frac{\omega_{*}^{2}-c r n_{*}^{2}}{\left(c r n_{*}^{2}+\omega_{*}^{2}\right) \omega_{*}^{2}}=\frac{2 \omega_{*}-r n_{*}}{\left(c r n_{*}^{2}+\omega_{*}^{2}\right) \omega_{*}} .
$$

Therefore,

$$
\begin{aligned}
\operatorname{sign}\left\{\left.\frac{d[\operatorname{Re} \xi(\tau)]}{d \tau}\right|_{\tau=\tau_{*}}\right\} & =\operatorname{sign}\left\{\left.\operatorname{Re}\left(\frac{d \xi}{d \tau}\right)^{-1}\right|_{\tau=\tau_{*}}\right\} \\
& =\operatorname{sign}\left\{2 \omega_{*}-r n_{*}\right\} .
\end{aligned}
$$

Then, from (7), we have the following results.

(i) Let $r-4 c=0$; then $\omega_{*}=\omega_{0}$, so that $\operatorname{sign}\left\{2 \omega_{*}-r n_{*}\right\}=$ 0 .

(ii) Let $r-4 c>0$.

If $\omega_{*}=\omega_{+}$, then $\operatorname{sign}\left\{2 \omega_{*}-r n_{*}\right\}=$ $\operatorname{sign}\left\{n_{*} \sqrt{r^{2}-4 c r}\right\}=+1$.

If $\omega_{*}=\omega_{-}$, then $\operatorname{sign}\left\{2 \omega_{*}-r n_{*}\right\}=$ $\operatorname{sign}\left\{-n_{*} \sqrt{r^{2}-4 c r}\right\}=-1$.

This completes the proof.

Bearing the above analysis in mind, we have that the root $\xi(\tau)$ of (2) crosses the imaginary axis from left to right at $\tau=$ $\tau_{j}^{+}$(because $d\left[\operatorname{Re} \xi\left(\tau_{j}^{+}\right)\right] / d \tau>0$ ) and from right to left at $\tau=$ $\tau_{j}^{-}$(because $d\left[\operatorname{Re} \xi\left(\tau_{j}^{-}\right)\right] / d \tau<0$ ), as $\tau$ increases.

Summarizing the above remarks and combining the lemmas, we have the following results on the distribution of roots of (2).

Theorem 5. For the system (1), the following statements hold true.

(1) If $r-4 c<0$, the equilibrium $\left(\kappa_{*}, n_{*}\right)=(\lambda / c, \lambda / c)$ is unstable for all $\tau \geq 0$.

(2) If $r-4 c>0$, we have two cases:

(a) the equilibrium $\left(\kappa_{*}, n_{*}\right)$ is unstable for all $\tau \geq$ 0 and undergoes a Hopf bifurcation at $\left(\kappa_{*}, n_{*}\right)$ when $\tau=\tau_{j}^{+}(j=0,1,2, \ldots)$;

(b) the equilibrium $\left(\kappa_{*}, n_{*}\right)$ is unstable for $\tau \in\left[0, \tau_{0}^{-}\right)$ and stable for $\tau>\tau_{0}^{-}$. Moreover, it undergoes $a$ Hopf bifurcation at $\left(\kappa_{*}, n_{*}\right)$ when $\tau=\tau_{j}^{-}(j=$ $0,1,2, \ldots)$.

Remark 6. In the previous theorem, the dynamics proprieties of the mathematical model are missing when $r-4 c=0$. Indeed, in this case, the transversality condition disappears. 


\section{Qualitative Analysis of the Hopf Bifurcation}

Following the normal form method and the center manifold theory [22], this section is concerned with the derivation of explicit formulas for determining the properties of the Hopf bifurcation (it exists when $r-4 c>0$, see Theorem 5 ) at critical value $\tau_{*}=\tau_{j}^{-}$or $\tau_{j}^{+}$, where $\lambda\left(\tau_{*}\right)=i \omega_{*}$.

In what follows, we denote by $C=C\left(\left[-\tau_{*}, 0\right], \mathbb{R}^{2}\right)$ the space of the continuous vector function

$$
v:\left[-\tau_{*}, 0\right] \longrightarrow \mathbb{R}^{2}
$$

and by $\widetilde{C}=C\left(\left[0, \tau_{*}\right], \mathbb{R}^{2}\right)$ the space of the continuous vector function

$$
v:\left[0, \tau_{*}\right] \longrightarrow \mathbb{R}^{2} \text {. }
$$

Setting $\mu=\tau-\tau_{*} \in \mathbb{R}$ and applying to the mathematical model (1) the change of variables

$$
\begin{aligned}
& u_{1}=\kappa-\kappa_{*}, \\
& u_{2}=n-n_{*},
\end{aligned}
$$

the model is equivalent to the functional differential equation system:

$$
\dot{u}(t)=L_{\mu}\left(u_{t}\right)+f\left(\mu, u_{t}\right),
$$

where

(i) $u=\left(u_{1}, u_{2}\right)$,

(ii) $u_{t}(\theta)=u(t+\theta)$ for $\theta \in\left[-\tau_{*}, 0\right]$,

(iii) $L_{\mu}: C \rightarrow \mathbb{R}^{2}$ reads as

$$
L_{\mu}(\varphi)=B_{1} \varphi(0)+B_{2} \varphi\left(-\tau_{*}\right) \text {, }
$$

where

$$
B_{1}=\left[\begin{array}{cc}
0 & c n_{*} \\
r n_{*} & 0
\end{array}\right], \quad B_{2}=\left[\begin{array}{cc}
0 & 0 \\
0 & -r n_{*}
\end{array}\right] \text {, }
$$

(iv) $f: \mathbb{R} \times C \rightarrow \mathbb{R}^{2}$ reads as

$$
f(\mu, \varphi)=\left[\begin{array}{l}
f_{1} \\
f_{2}
\end{array}\right],
$$

where

$$
f_{1}=c \varphi_{1}(0) \varphi_{2}(0), \quad f_{2}=r \varphi_{1}(0) \varphi_{2}(0)-r \varphi_{2}(0) \varphi_{2}\left(-\tau_{*}\right) .
$$

Lemma 7. Let $L_{\mu}(\varphi)$ be the vector function defined by (20). Then, there exists a $2 \times 2$ matrix-valued function of bounded variation $\eta(\theta, \mu)$, for $\theta \in\left[-\tau_{*}, 0\right]$, such that

$$
L_{\mu} \varphi=\int_{-\tau_{*}}^{0} \varphi(\theta) d \eta(\theta, \mu) .
$$

Proof. The proof is obtained by applying the Riesz representation theorem. Indeed, we may take

$$
\eta(\theta, \mu)=B_{1} \delta(\theta)+B_{2} \delta\left(\theta+\tau_{*}\right),
$$

where $\delta(\theta)$ is the Dirac delta function.

Definition 8. Let $\eta(\theta, \mu)$ be the $2 \times 2$ matrix-valued function $\eta(\theta, \mu)$ of bounded variation of Lemma 7 . For $\varphi \in C$, one defines the following operators:

$$
\begin{gathered}
A(\mu)(\varphi):= \begin{cases}\frac{d \varphi(\theta)}{d \theta}, & \theta \in\left[-\tau_{*}, 0\right), \\
\int_{-\tau_{*}}^{0} d \eta(s, \mu) \varphi(s), & \theta=0,\end{cases} \\
R(\mu)(\varphi):= \begin{cases}0, & \theta \in\left[-\tau_{*}, 0\right), \\
f(\mu, \varphi), & \theta=0 .\end{cases}
\end{gathered}
$$

Remark 9. A straightforward analysis shows that the system (19) is equivalent to

$$
\dot{u}(t)=A(\mu) u_{t}+R(\mu) u_{t},
$$

where $A(\mu)(\varphi)$ and $R(\mu)(\varphi)$ are the operators (26) and (27), respectively, and $u_{t}=u(t+\theta)$, for $\theta \in\left[-\tau_{*}, 0\right]$.

Definition 10. Let $\eta(\theta, \mu)$ be the $2 \times 2$ matrix-valued function $\eta(\theta, \mu)$ of bounded variation of Lemma 7 . For $\psi \in \widetilde{C}=$ $C\left(\left[0, \tau_{*}\right], \mathbb{R}^{2}\right)$, one defines the following operator:

$$
A^{*}(\mu) \psi(s)= \begin{cases}-\frac{d \psi(s)}{d s}, & s \in\left(0, \tau_{*}\right], \\ \int_{-\tau_{*}}^{0} d \eta(\zeta, \mu) \psi(-\zeta), & s=0,\end{cases}
$$

and one defines the following bilinear form:

$$
\begin{aligned}
\langle\psi(s), \varphi(\theta)\rangle= & \bar{\psi}(0) \varphi(0) \\
& -\int_{\theta=-\tau_{*}}^{0} \int_{\sigma=0}^{\theta} \bar{\psi}(\sigma-\theta) d \eta(\theta) \varphi(\sigma) d \sigma,
\end{aligned}
$$

where $\eta(\theta)=\eta(\theta, 0)$.

Remark 11. It is easy to show that $A(0)$ and $A^{*}(0)$ are adjoint operators. We know that $\pm i \omega_{*}$ is an eigenvalue of $A(0)$; then $\pm i \omega_{*}$ is also an eigenvalue of $A^{*}(0)$.

Let $q(\theta)$ and $q^{*}(s)$ be the eigenvectors for $A(0)$ and $A^{*}(0)$ corresponding to $i \omega_{*}$ and $-i \omega_{*}$. Assume that $q(\theta)=$ $q(0) e^{i \omega_{*} \theta}=\left(1, \alpha_{1}\right) e^{i \omega_{*} \theta}$, with $\alpha_{1} \in \mathbb{C}$, is the eigenvector of $A(0)$ corresponding to $i \omega_{*}$. From $A(0) q(\theta)=i \omega_{*} q(\theta)$, we have $\left(B_{1}-i \omega_{*} I+B_{2} e^{-i \omega_{*} \theta}\right) q(0)=0$, so that we can evaluate $\alpha_{1}$. Similarly, we assume $q^{*}(r)=q^{*}(0) e^{i \omega_{*} r}=D\left(\alpha_{2}, 1\right) e^{i \omega_{*} r}$, where $\alpha_{2}$ and $D$ are complex values, and get $\left(B_{1}^{T}+i \omega_{*} I+\right.$ $\left.B_{2}^{T} e^{-i \omega_{*} \theta}\right) q^{*}(0)=0$. In this way, we can find $\alpha_{2}$ with the value of $D$ chosen so that $\left\langle q^{*}(s), q(\theta)\right\rangle=1$. 
We will use now the theory by Hassard et al. [22] to compute the coordinates describing the center manifold $\mathscr{C}$ at $\mu=0$. Let $u_{t}$ be a solution of (28) with $\mu=0$ and

$$
z(t):=\left\langle q^{*}, u_{t}\right\rangle, \quad W(t, \theta):=u_{t}(\theta)-2 \operatorname{Re}[z(t) q(\theta)] .
$$

On the center manifold $\mathscr{C}$, one has

$$
W(t, \theta)=W(z(t), \bar{z}(t), \theta),
$$

where

$$
\begin{aligned}
W(t, \theta) & =W(z(t), \bar{z}(t), \theta) \\
& =W_{20}(\theta) \frac{z^{2}}{2}+W_{11}(\theta) z \bar{z}+W_{02}(\theta) \frac{\bar{z}^{2}}{2}+\cdots,
\end{aligned}
$$

and $z$ and $\bar{z}$ are local coordinates for the center manifold $\mathscr{C}$ in the direction of $q$ and $\bar{q}$. For the solution $u_{t} \in \mathscr{C}$ of (28), since $\mu=0$, we get

$$
\dot{z}(t)=i \omega\left(\tau_{*}\right) z+\bar{q}^{*}(0) f_{0}(z, \bar{z}),
$$

where

$$
f_{0}(z, \bar{z})=f(0, W(z, \bar{z}, 0)+2 \operatorname{Re}[z(t) q(0)]),
$$

and $W(z, \bar{z}, 0)$ is given by (32). We rewrite this equation $\dot{z}(t)=$ $i \omega_{*} z+g(z, \bar{z})$, where

$$
\begin{aligned}
g(z, \bar{z}) & =\bar{q}^{*}(0) f_{0}(z, \bar{z}) \\
& =g_{20} \frac{z^{2}}{2}+g_{11} z \bar{z}+g_{02} \frac{\bar{z}^{2}}{2}+g_{21} \frac{z^{2} \bar{z}}{2}+\cdots .
\end{aligned}
$$

Since $u_{t}(\theta)=W(t, \theta)+z q(\theta)+\bar{z} \bar{q}(\theta)$, substituting into $f_{0}$, we find

$$
f_{0}=f_{z^{2}} \frac{z^{2}}{2}+f_{z \bar{z}} z \bar{z}+f_{\bar{z}^{2}} \frac{\bar{z}^{2}}{2}+f_{z^{2} \bar{z}} \frac{z^{2} \bar{z}}{2}+\cdots
$$

Comparing the coefficients between (36) and (37), we obtain

$$
\begin{array}{ll}
g_{20}=\bar{q}^{*}(0) f_{z^{2}}, & g_{02}=\bar{q}^{*}(0) f_{\bar{z}^{2}}, \\
g_{11}=\bar{q}^{*}(0) f_{z \bar{z}}, & g_{21}=\bar{q}^{*}(0) f_{z^{2} \bar{z}} .
\end{array}
$$

We remark that $g_{21}$, will depend on $W_{20}(\theta)$ and $W_{11}(\theta)$. Hence, in order to determine $g_{21}$, we need to compute them. By (28) and (36), we have

$$
\begin{aligned}
\dot{W} & =\dot{u}_{t}-\dot{z} q-\dot{\bar{z}} \bar{q} \\
& = \begin{cases}A(0) W-2 \operatorname{Re}\left[\bar{q}^{*}(0) f_{0} q(\theta)\right], & \theta \in\left[-\tau_{*}, 0\right), \\
A(0) W-2 \operatorname{Re}\left[\bar{q}^{*}(0) f_{0} q(0)\right]+f_{0}, & \theta=0,\end{cases} \\
& :=A(0) W+H(z, \bar{z}, \theta),
\end{aligned}
$$

where

$$
H(z, \bar{z}, \theta)=H_{20}(\theta) \frac{z^{2}}{2}+H_{11}(\theta) z \bar{z}+H_{02}(\theta) \frac{\bar{z}^{2}}{2}+\cdots .
$$

Expanding the above series and comparing the corresponding coefficients of $z^{2}, z \bar{z}$, and $\bar{z}^{2}$, we obtain

$$
\begin{gathered}
{\left[A(0)-2 i \omega_{*}\right] W_{20}(\theta)=-H_{20}(\theta),} \\
A(0) W_{11}(\theta)=-H_{11}(\theta) .
\end{gathered}
$$

From (39), for $\theta \in\left[-\tau_{*}, 0\right)$, we can get

$$
H(z, \bar{z}, \theta)=2 \operatorname{Re}\left[\bar{q}^{*}(0) f_{0} q(\theta)\right]=-q(\theta)-\bar{g} \bar{q}(\theta) .
$$

Comparing the coefficients with (40), we obtain

$$
\begin{aligned}
& H_{20}(\theta)=-g_{20} q(\theta)-\bar{g}_{02} \bar{q}(\theta), \\
& H_{11}(\theta)=-g_{11} q(\theta)-\bar{g}_{11} \bar{q}(\theta) .
\end{aligned}
$$

Combining (41) and (43), we derive

$$
\begin{gathered}
\dot{W}_{20}(\theta)=2 i \omega_{*} W_{20}(\theta)-H_{20}(\theta), \\
\dot{W}_{11}(\theta)=-H_{11}(\theta) .
\end{gathered}
$$

Therefore,

$$
\begin{gathered}
W_{20}(\theta)=-\frac{g_{20}}{i \omega_{*}} q(0) e^{i \omega_{*} \theta}-\frac{\bar{g}_{02}}{3 i \omega_{*}} \bar{q}(0) e^{-i \omega_{*} \theta}+E_{1} e^{2 i \omega_{*} \theta}, \\
W_{11}(\theta)=\frac{g_{11}}{i \omega_{*}} q(0) e^{i \omega_{*} \theta}-\frac{\bar{g}_{11}}{i \omega_{*}} \bar{q}(0) e^{-i \omega_{*} \theta}+E_{2},
\end{gathered}
$$

where $\left(E_{1}, E_{2}\right)$ is a constant vector to be determined. From (31), we find

$$
\begin{gathered}
\int_{-\tau_{*}}^{0} d \eta(\theta) W_{20}(\theta)=2 i \omega_{*} W_{20}(\theta)-H_{20}(\theta) \\
\int_{-\tau_{*}}^{0} d \eta(\theta) W_{11}(\theta)=-H_{11}(\theta) .
\end{gathered}
$$

From (31) again, for $\theta=0$, we see that

$$
\begin{aligned}
& H_{20}(0)=-g_{20} q(0)-\bar{g}_{02} \bar{q}(0)+f_{z^{2}}, \\
& H_{11}(0)=-g_{11} q(0)-\bar{g}_{11} \bar{q}(0)+f_{z \bar{z}} .
\end{aligned}
$$

Substituting (46) and (50) into (48) and observing that

$$
\begin{gathered}
{\left[i \omega_{*} I-\int_{-\tau_{*}}^{0} e^{i \omega_{*} \theta} d \eta(\theta)\right] q(0)=0,} \\
{\left[-i \omega_{*} I-\int_{-\tau_{*}}^{0} e^{-i \omega_{*} \theta} d \eta(\theta)\right] \bar{q}(0)=0,}
\end{gathered}
$$

we have

$$
\left[2 i \omega_{*}-\int_{-\tau_{*}}^{0} e^{2 i \omega_{*} \theta} d \eta(\theta)\right] E_{1}=f_{z^{2}}
$$

and then we get $E_{1}$. Similarly for $E_{2}$, we have

$$
\left[\int_{-\tau_{*}}^{0} d \eta(\theta)\right] E_{2}=f_{z \bar{z}}
$$


Thus, we can calculate all the four coefficients $g_{i j}$ and so can determine the following quantities, which are required for the analysis of Hopf bifurcation:

$$
\begin{gathered}
C_{1}(0)=\frac{i}{2 \omega_{*}}\left[g_{11} g_{20}-2\left|g_{11}\right|^{2}-\frac{\left|g_{02}\right|^{2}}{3}\right]+\frac{g_{21}}{2}, \\
\mu_{2}=-\frac{\operatorname{Re}\left\{C_{1}(0)\right\}}{\operatorname{Re}\left\{\lambda^{\prime}\left(\tau_{*}\right)\right\}}, \quad \beta_{2}=2 \operatorname{Re}\left\{C_{1}(0)\right\}, \\
T_{2}=-\frac{\operatorname{Im}\left\{C_{1}(0)\right\}+\mu_{2} \operatorname{Im}\left\{\lambda^{\prime}\left(\tau_{*}\right)\right\}}{\omega_{*}} .
\end{gathered}
$$

The periodic solutions and their stabilities can be analyzed with the aid of the normal form parameters $C_{1}(0), \mu_{2}, \beta_{2}$, and $T_{2}$. In particular, $\mu_{2}$ allows us to determine the quality of the Hopf bifurcation (supercritical or subcritical); $\beta_{2}$ determines the stability of the bifurcating periodic solutions; $T_{2}$ determines the period of the bifurcating periodic solutions.

Theorem 12. Let $\left(\kappa_{*}, n_{*}\right)$ be the equilibrium point of the model (1).

(1) If $\mu_{2}>0$, then the Hopf bifurcation of system (1) at the equilibrium point $\left(\kappa_{*}, n_{*}\right)$, when $\tau=\tau_{*}$, is supercritical; if $\mu_{2}<0$, the Hopf bifurcation is subcritical.

(2) The bifurcating periodic solutions are locally asymptotically stable if $\beta_{2}<0$ and unstable if $\beta_{2}>0$.

(3) The period of the bifurcating periodic solutions increases if $T_{2}>0$ and decreases if $T_{2}<0$.

\section{Applications and Research Perspectives}

The mathematical framework proposed in the present paper can be used in applications. Indeed, the framework can be applied for the modeling of biological system, such as the competition between cancer cells and immune system cells; see paper [23]. The logistic growth term models the maximum cancer cells population size that can be sustained during proliferation. Moreover applications refer to economic growth theory; see [24-26]. the logistic growth is usually attributed to resources per individual becoming scarcer as population size increases. In the case of human population growth, however, it is natural to assume that technology, social organization, and other aspects of culture have allowed humans to increase the environment's carrying capacity.

The mathematical framework proposed in this paper is certainly worthy of future research concerning both its qualitative analysis and the application to modeling complex systems in applied sciences. Specifically, analytical investigations can be addressed to the existence of limit cycles [2729] such as the contents of the paper [30]. Moreover, it is a research perspective to analyze the stability and Hopf bifurcation of the model (1) when time delay is inserted also in the $\kappa$ function.

Finally, the mathematical framework (1) can be generalized in order to take into account strategies performed by the elements of the system (especially in the context of biological systems) by using methods of thermostated kinetic equations [31, 32] that allow the possibility to reach a stationary state [33]. Moreover, this approach permits us to perform asymptotic limits linking ordinary differential equations with kinetic and continuum mechanics approaches [34].

\section{Acknowledgment}

The first author acknowledges the support of the FIRB project RBID08PP3J-Metodi Matematici e Relativi Strumenti per la Modellizzazione e la Simulazione della Formazione di tumori, Competizione con il Sistema Immunitario, e Conseguenti Suggerimenti Terapeutici.

\section{References}

[1] R. Eftimie, J. L. Bramson, and D. J. D. Earn, "Interactions between the immune system and cancer: a brief review of nonspatial mathematical models," Bulletin of Mathematical Biology, vol. 73, no. 1, pp. 2-32, 2011.

[2] N. F. Britton, Reaction-Diffusion Equations and Their Applications to Biology, Academic Press, London, Uk, 1986.

[3] F. Mollica, L. Preziosi, and K. R. Rajagopal, Eds., Modeling of Biological Materials, Modeling and Simulation in Science, Engineering and Technology, Birkhäuser, Boston, Mass, USA, 2007.

[4] C. Bianca, "Thermostatted kinetic equations as models for complex systems in physics and life sciences," Physics of Life Reviews, vol. 9, pp. 359-399, 2012.

[5] C. T. H. Baker, G. A. Bocharov, and C. A. H. Paul, "Mathematical modelling of the interleukin-2 T-cell system: a comparative study of approaches based on ordinary and delay differential equations," Journal of Theoretical Medicine, vol. 2, pp. 117-128, 1997.

[6] M. Badii and A. Schiaffino, "Asymptotic behaviour of positive solutions of periodic delay logistic equations," Journal of Mathematical Biology, vol. 14, no. 1, pp. 95-100, 1982.

[7] J. Bélair and S. A. Campbell, "Stability and bifurcations of equilibria in a multiple-delayed differential equation," SIAM Journal on Applied Mathematics, vol. 54, no. 5, pp. 1402-1424, 1994.

[8] J. Blair, M. C. Mackey, and J. M. Mahay, "Age-structured and two delay models for erythropoiesis," Mathematical Biosciences, vol. 128, pp. 317-346, 1995.

[9] H. I. Freedman and K. Gopalsamy, "Global stability in timedelayed single-species dynamics," Bulletin of Mathematical Biology, vol. 48, no. 5-6, pp. 485-492, 1986.

[10] S. A. Gourley and M. A. J. Chaplain, "Travelling fronts in a foodlimited population model with time delay," Proceedings of the Royal Society of Edinburgh A, vol. 132, no. 1, pp. 75-89, 2002.

[11] Y. Kuang, Delay Differential Equations with Applications in Population Dynamics, vol. 191 of Mathematics in Science and Engineering, Academic Press, Boston, Mass, USA, 1993.

[12] C. Bianca, M. Ferrara, and L. Guerrini, "Hopf bifurcations in a delayed-energy-based model of capital accumulation," Applied Mathematics \& Information Sciences, vol. 7, no. 1, pp. 139-143, 2013.

[13] C. Bianca, M. Ferrara, and L. Guerrini, "The Cai model with time delay: existence of periodic solutions and asymptotic analysis," Applied Mathematics \& Information Sciences, vol. 7, no. 1, pp. 21-27, 2013. 
[14] C. Bianca and L. Guerrini, "On the Dalgaard-Strulik model with logistic population growth rate and delayed-carrying capacity," Acta Applicandae Mathematicae, vol. 128, pp. 39-48, 2013.

[15] P. F. Verhulst, "Notice sur la loi que la population suit dans son accroissement," Correspondance Mathématique et Physique, vol. 10, pp. 113-121, 1838.

[16] G. E. Hutchinson, "Circular causal systems in ecology," Annals of the New York Academy of Sciences, vol. 50, no. 4, pp. 221-246, 1948.

[17] M. Kremer, "Population growth and technological change: one million BC to 1990," Quarterly Journal of Economics, vol. 108, no. 3, pp. 681-716, 1993.

[18] A. Korotayev, "A compact macromodel of world system evolution," Journal of World-Systems Research, vol. 11, pp. 79-93, 2005.

[19] A. R. Rogers, "Genetic evidence for a pleistocene population explosion," Evolution, vol. 49, no. 4, pp. 608-615, 1995.

[20] O. Bar-Yosef, "The Upper Paleolithic revolution," Annual Review of Anthropology, vol. 31, pp. 363-393, 2002.

[21] J. E. Cohen, "Population growth and earth's human carrying capacity," Science, vol. 269, no. 5222, pp. 341-346, 1995.

[22] B. Hassard, D. Kazarino, and Y. Wan, Theory and Application of Hopf Bifurcation, Cambridge, UK, 1981.

[23] C. Bianca and M. Pennisi, "The triplex vaccine effects in mammary carcinoma: a nonlinear model in tune with SimTriplex," Nonlinear Analysis: Real World Applications, vol. 13, no. 4, pp. 1913-1940, 2012.

[24] R. M. Solow, "A contribution to the theory of economic growth," Quarterly Journal of Economics, vol. 70, pp. 65-94, 1956.

[25] T. W. Swan, "Economic growth and capital accumulation," Economic Record, vol. 32, pp. 334-361, 1956.

[26] L. Guerrini, "The Solow-Swan model with a bounded population growth rate," Journal of Mathematical Economics, vol. 42, no. 1, pp. 14-21, 2006.

[27] H. Poincaré, "Mémoire sur les courbes définies par une équation différentialle. I, II," Journal de Mathématiques Pures et Appliquées, vol. 7, pp. 375-422, 1881.

[28] I. Bendixson, "Sur les courbes définies par des équations différentielles," Acta Mathematica, vol. 24, no. 1, pp. 1-88, 1901.

[29] Yu. S. Ilyashenko, "Finiteness theorems for limit cycles," Uspekhi Matematicheskikh Nauk, vol. 45, no. 2, pp. 143-200, 1990.

[30] L. V. Ballestra, L. Guerrini, and G. Pacelli, "Stability switches and Hopf bifurcation in a kaleckian model of business cycle," Abstract and Applied Analysis, vol. 2013, Article ID 689372, 8 pages, 2013.

[31] C. Bianca, "Modeling complex systems by functional subsystems representation and thermostatted-KTAP methods," Applied Mathematics \& Information Sciences, vol. 6, pp. 495499, 2012.

[32] C. Bianca, "Controllability in hybrid kinetic equations modeling nonequilibrium multicellular systems," The Scientific World Journal, vol. 2013, Article ID 274719, 6 pages, 2013.

[33] C. Bianca, "Existence of stationary solutions in kinetic models with Gaussian thermostats," Mathematical Methods in the Applied Sciences, vol. 36, pp. 1768-1775, 2013.

[34] A. Bellouquid and C. Bianca, "Modelling aggregation-fragmentation phenomena from kinetic to macroscopic scales," Mathematical and Computer Modelling, vol. 52, no. 5-6, pp. 802-813, 2010 . 


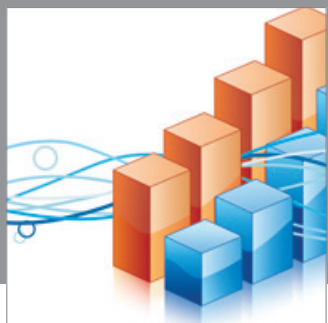

Advances in

Operations Research

mansans

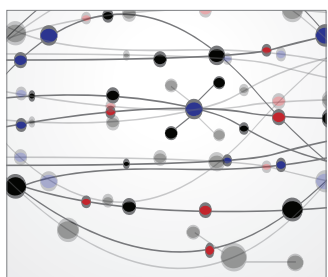

The Scientific World Journal
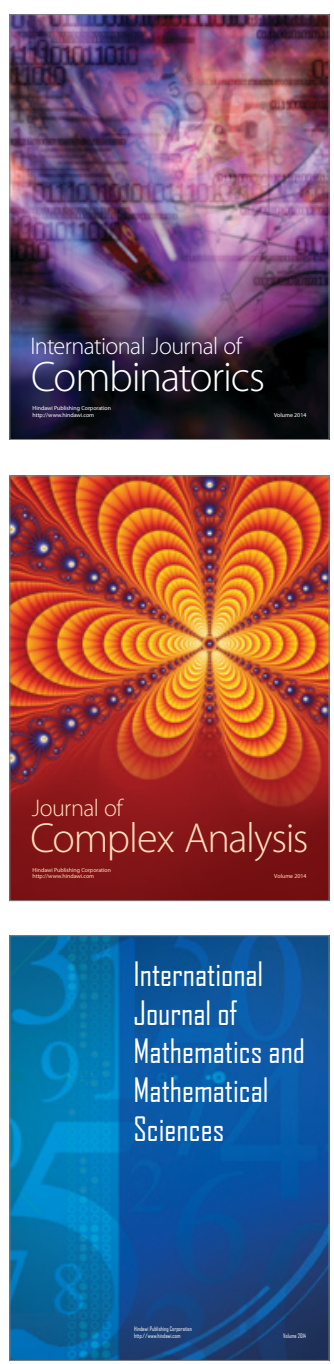
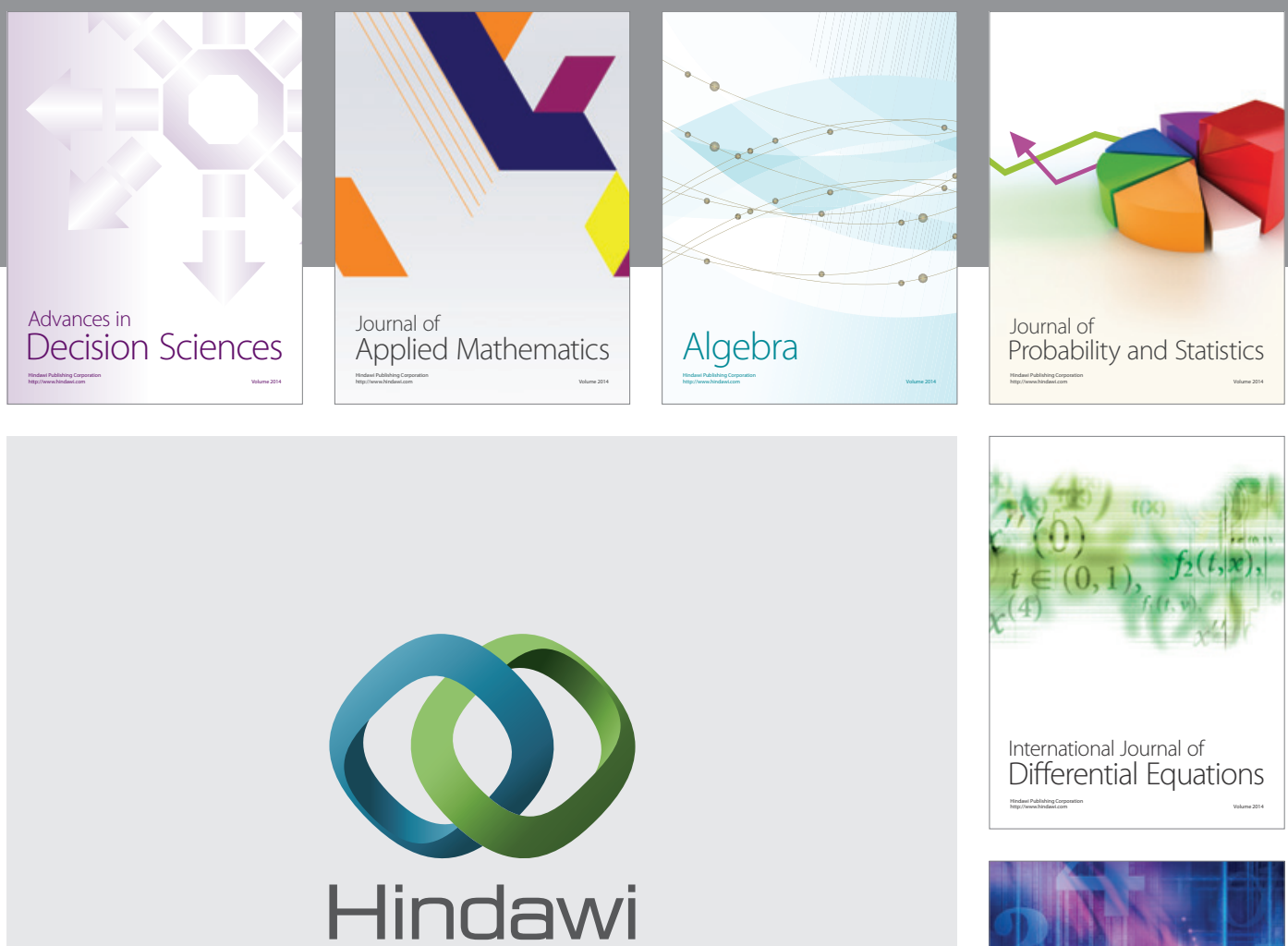

Submit your manuscripts at http://www.hindawi.com
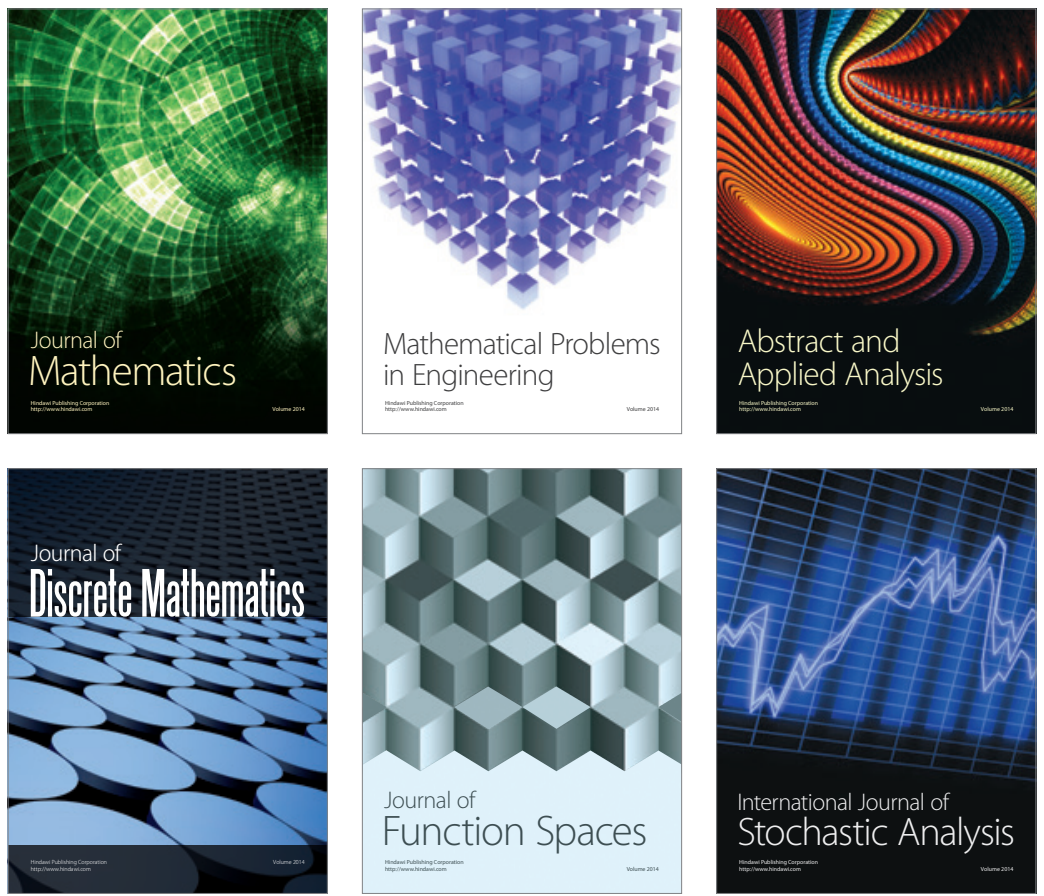

Journal of

Function Spaces

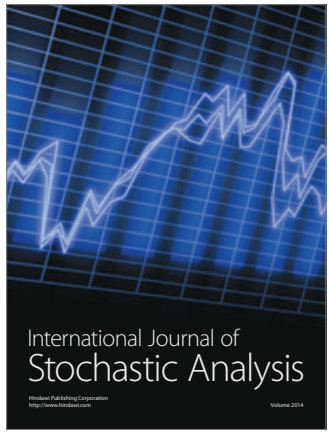

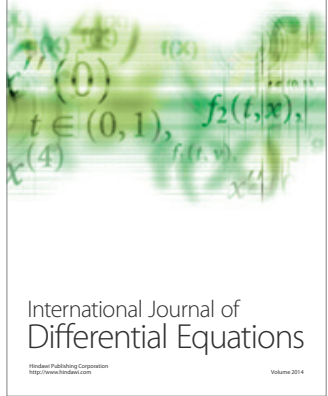
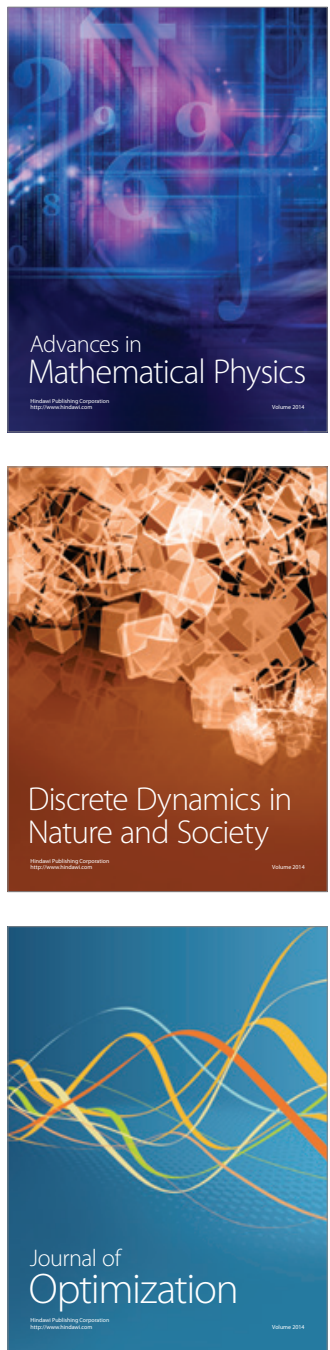